\title{
Technical demands of different playing positions in the UEFA Champions League
}

\section{Qing Yi, Hong Jia, Hongyou Liu \& Miguel Ángel Gómez}

To cite this article: Qing Yi, Hong Jia, Hongyou Liu \& Miguel Ángel Gómez (2018): Technical demands of different playing positions in the UEFA Champions League, International Journal of Performance Analysis in Sport, DOI: 10.1080/24748668.2018.1528524

To link to this article: https://doi.org/10.1080/24748668.2018.1528524

册 Published online: 08 Oct 2018.

Submit your article to this journal $\pi$

View Crossmark data \lceil 


\title{
Technical demands of different playing positions in the UEFA Champions League
}

\author{
Qing Yi (iD), Hong Jia ${ }^{\mathrm{b}}$, Hongyou Liu (D) and Miguel Ángel Gómez (D) ${ }^{\mathrm{a}}$ \\ a Faculty of Physical Activity and Sport Sciences, Technical University of Madrid, Madrid, Spain; \\ bDepartment of Physical Education, Guangdong University of Finance, Guangzhou, China; 'School of \\ Physical Education \& Sports Science, South China Normal University, Guangzhou, Guangdong, China
}

\begin{abstract}
This study aimed to identity the technical demands of different playing positions controlling the effects of situational variables in the UEFA Champions League from a long-term perspective. Data of 18 technical performance-related match actions and events achieved by 1,990 out-field players (classified into five positional roles) in 1,000 matches played in the UEFA Champions League from season 2009/2010 to 2016/2017 were collected. Generalised mixed linear modelling was employed taking the value of each of the 18 technical performance-related variables as the dependent variable, and taking the classified 5 positions as the predictor variable. Uncertainty in the true effects of the predictors was evaluated using non-clinical magnitude-based inference. Results showed that the differences between central defenders and forwards were biggest while central defenders and full backs presented the smallest difference. The performances of midfielders in variables related to passing and organising were worse than expected and wide midfielders showed relative better performances than central midfielders in passing and organising. Meanwhile, defenders, especially central defenders, achieved good performance in variables related to passing and organising. Forwards played an important role in the aspects of goal scoring and organising, they also participated in the initial defending process.
\end{abstract}

\section{ARTICLE HISTORY}

Received 21 August 2018

Accepted 23 September 2018

\section{KEYWORDS}

Technical demand; situational variable; modelling; match analysis; soccer; football

\section{Introduction}

Match performance of football is the result of the interaction of technical, tactical and physical activities presented by players within a match (Bush, Barnes, Archer, Hogg, \& Bradley, 2015; Carling, 2010; Dellal et al., 2008). Every player fulfils specific tasks and undertakes associated functions according to his/her playing position in the game, in order to achieve a successful performance, and eventually to win a match (Hughes et al., 2012). Hence, the positional difference should not be disregarded when evaluating players' match performance (Butterworth, O’Donoghue, \& Cropley, 2013) and the individualised criterion is needed to identify the characteristics and demands of each playing position, otherwise a wealth of valuable information might be masked which

CONTACT Hongyou Liu szu.youyou@hotmail.com E School of Physical Education \& Sports Science, South China Normal University, 510006, Guangzhou, China 
may lead to a restriction on the understanding of players' match performance (LagoPeñas, Casais, Dellal, Rey, \& Domínguez, 2011a).

There have been many studies conducted to identify the characteristics and demands of individual roles within a football team framework (Bloomfield, Polman, \& O’Donoghue, 2007; Boone, Vaeyens, Steyaert, Bossche, \& Bourgois, 2012; Dellal, Wong, Moalla, \& Chamari, 2010; Di Salvo et al., 2007; Mohr, Krustrup, \& Bangsbo, 2005; Reilly, Bangsbo, \& Franks, 2000). Most of the previous research is mainly concerning the physical demands or anthropometric and physiological characteristics of different positional roles based on time-motion analysis. It has been reported that midfielders covered significantly greater total distance compared with players of any other position and even performed more high-intensity running than central defenders and forwards. Full backs and forwards showed higher sprinting ability and better agility, while central defenders covered the shortest total distance and presented the least sprinting time. Moreover, Reilly et al. (2000) reported that fullbacks and midfielders showed the best performance in the intermittent exercise test and have the highest maximal oxygen intakes. On the contrary, midfielders appeared to have the lowest muscle strength.

However, there has been limited research investigating the position-specific technical match demands from an empirical perspective, even though technical indicators have been considered as better predictors of football match success (Lago-Peñas, LagoBallesteros, \& Rey, 2011b) and technical skills have been counted as a main component in football talent identification and development systems (Vaeyens, Lenoir, Williams, \& Philippaerts, 2008). Hughes et al. (2012) and Wiemeyer (2017) reported the technical demands of different playing positions in a qualitative way, the typical technical features and technical requirements of each positional role were identified and compared whereas limited systematic quantitative data were included. Taylor, Mellalieu, and James (2004) developed a notational analysis system to construct inter-positional and intra-positional behavioural and performance profiles, but the post-hoc test was not used in the non-parametric analysis hence it failed to identify the differences between any two playing positions. Vale et al. (2009) conducted a more quantitative research, which concluded that there were no significant differences in passing abilities of young players (17-18 years old) when considering playing positions. However, the data of this study was based on three tests of technical skills rather than the match statistics from official football matches. Dellal et al. (2010) identified the positional demands from both physical and technical aspects in the French First League, main findings include: midfielders showed the greatest total distance covered; forwards covered the greatest distance in sprinting but lost the greatest number of duels, while central defenders and full backs won the highest number of duels, and central defenders also showed the lowest values of successful passes. Nevertheless, this study failed to address the repeated-measured data of players who played in multiple matches. Furthermore, position-specific technical demands identified in these studies were not controlled for the effects of situational factors (e.g. team and opponent strength, match location, competition phase, etc.) which have already been proved to largely affect the technical performance of football players from a behaviour level (Gómez, Lago, \& Pollard, 2013; Lago-Peñas \& Lago-Ballesteros, 2011; Liu, Yi, Giménez, Gómez, \& Lago-Peñas, 2015). In addition, the sampling issues have been disregarded in these studies. The small 
number of players may fail to produce meaningful typical performance of each playing position (Taylor et al., 2004), while the mean values of indicators can yield valuable information about position specific demands based on a large number of players observed in each playing position (Reilly et al., 2000).

Consequently, the current study aims to quantitatively identify the position-specific technical demands of elite football players in the UEFA Champions League using one of the largest samples published to date. The generalised linear mixed modelling is employed to deal appropriately with repeated-measure data of players from multiple matches in a long-term perspective. Situational variables of competition stage, match location, team and opponent strength were controlled by the modelling in order to define sets of key performance indicators for each position.

\section{Methods}

\subsection{Data and reliability}

Technical performance-related match data of all the 1,000 games played in the UEFA Champions League from season 2009/2010 to 2016/2017 were collected for analysis. Data resource is a public-accessed football statistic website named "whoscored.com" (https://www.whoscored.com), which uses the original data from OPTA Sportsdata company. The inter-operator reliability of the data-coding system when collecting information of football players' match actions and events has been tested to be at an acceptable level (Liu, Hopkins, Gómez, \& Molinuevo, 2013). This study maintains the anonymity of the players following European data protection law and is officially approved by the ethics committee of the local university.

\subsection{Sample and variables}

Sample of the current study consisted of all players who started and played at least one entire match in the UEFA Champions Leagued during the eight seasons of 2009/2010$2016 / 2017$. Substitutes and the players they substituted were excluded. And due to the specificity of the position of goalkeeper, match data of players from this position were excluded as well. Hence, the sample finally came to 1,990 players who generated 14,437 full match observations.

Players in the sample were divided into 5 groups according to their playing positions at the start of each game (Bush et al., 2015; Liu, Gómez, Gonçalves, \& Sampaio, 2016a): Central Defender (CD, $n=3632$ match observations), Full Back (FB, $n=3429$ ), Central Midfielder (CM, $n=4044)$, Wide Midfielder (WM, $n=1565$ ), and Forward (FW, $n=1767)$.

Based on previous literature (Castellano, Casamichana, \& Lago, 2012; Lago-Peñas \& Lago-Ballesteros, 2011; Lago-Peñas, Lago-Ballesteros, Dellal, \& Gómez, 2010; Liu et al., 2016a; Liu, Hopkins, \& Gómez, 2016b; Liu et al., 2015; Mao, Peng, Liu, \& Gómez, 2016), count numbers of 18 technical performance-related match events and actions were selected to act as variables identifying the technical match performance of players. The grouping and operational definitions of these variables can be found in Table 1 (Liu et al., 2016a, 2016b, 2013; Mao et al., 2016). 
Table 1. Selected performance-related match events and actions.

\begin{tabular}{|c|c|}
\hline Groups & Event or action: operational definition \\
\hline $\begin{array}{l}\text { Variables related to goal } \\
\text { scoring }\end{array}$ & $\begin{array}{l}\text { Shot: an attempt to score a goal, made with any (legal) part of the body, either on } \\
\text { or off target } \\
\text { Shot on target: an attempt to goal which required intervention to stop it going in or } \\
\text { resulted in a goal/shot which would go in without being diverted }\end{array}$ \\
\hline $\begin{array}{l}\text { Variables related to passing } \\
\text { and organising }\end{array}$ & $\begin{array}{l}\text { Touch: a sum of count values of all actions and events where a player touches the } \\
\text { ball } \\
\text { Pass: an intentional played ball from one player to another } \\
\text { Pass accuracy (\%): successful passes as a proportion of total passes } \\
\text { Key pass: the final pass or cross leading to the recipient of the ball having an } \\
\text { attempt at goal without scoring } \\
\text { Cross: any ball sent into the opposition team's area from a wide position } \\
\text { Long ball: an attempted pass of } 25 \text { yards or more } \\
\text { Through ball: a pass that split the last line of defence and plays the teammate } \\
\text { through on goal } \\
\text { Aerial won: two players competing for a ball in the air, for it to be an aerial duel } \\
\text { both players must jump and challenge each other in the air and have both feet } \\
\text { off the ground. The player who wins the duel gets the Aerial won, and the player } \\
\text { who does not gets an Aerial lost. } \\
\text { Dribble: a dribble is an attempt by a player to beat an opponent in possession of } \\
\text { the ball. OPTA also log attempted dribbles where the player overruns the ball. } \\
\text { Fouled: where a player is fouled by an opponent } \\
\text { Dispossessed: when a player is tackled without attempting to dribble past his } \\
\text { opponent }\end{array}$ \\
\hline Variables related to defending & $\begin{array}{l}\text { Tackle: the action of gaining possession from an opposition player who is in } \\
\text { possession of the ball } \\
\text { Interception: a player intercepts a pass with some movement or reading of the play } \\
\text { Clearance: attempt made by a player to get the ball out of the danger zone, when } \\
\text { there is pressure (from opponents) on him to clear the ball } \\
\text { Foul: any infringement that is penalised as foul play by a referee } \\
\text { Yellow card: where a player was shown a yellow card by the referee for reasons of } \\
\text { foul, persistent infringement, hand ball, dangerous play, etc. }\end{array}$ \\
\hline
\end{tabular}

\subsection{Procedure and statistical analysis}

A generalised mixed linear model was realised with Proc Glimmix in the University Edition of Statistical Analysis System (version SAS Studio 3.6). A random effect for player identity was used to account for repeated measurement on the players. The fixed effects estimated the effect of the playing position, as well as the effect of game location, competition phase, team and opposition strength. Separate Poisson regressions were run in the model taking the value of each of the 18 technical performance-related variables as the dependent variable.

Playing position, game location and competition phase were all included as nominal predictor variables in the model. Playing position was with five levels (CD, FB, CM, WM and FW), game location was with three levels (home, away and neutral), and competition phase was with two levels (group stage and knock-out stage). The effect of team strength and opponent strength was estimated by including the difference in the log of the end-ofseason UEFA club coefficient as a predictor (Liu et al., 2015; Phillips \& Hopkins, 2017).

Uncertainty in the true effects of the predictors was evaluated using non-clinical magnitude-based inference as implemented in the spreadsheet accompanying the package of materials for generalised mixed modelling with SAS Studio (Hopkins, 2016). Observed magnitudes and their confidence limits were expressed in standardised units, whereby the difference in means was divided by the observed between-player standard 
deviation (SD) derived from the mixed model, then evaluated qualitatively with the following scale: $<0.2$ trivial, $0.2-0.6$ small, 0.6-1.2 moderate, 1.2-2.0 large, $>2.0$ very large (Hopkins, Marshall, Batterham, \& Hanin, 2009). Effects were deemed clear if the 90\% confidence interval (CI) did not include positive and negative substantial values simultaneously. Clear effects were reported with a qualitative likelihood that the true effect was either substantial or trivial (whichever probability was greater) using the following scale: $<0.5 \%$ most unlikely, $0.5-5 \%$ very unlikely, $5-25 \%$ unlikely, $25-75 \%$ possibly, 75-95\% likely, 95-99.5\% very likely, > 99.5\% most likely (Hopkins et al., 2009).

\section{Results}

Mean and between-player standard deviation of the 18 technical performance-related match actions and events of players from five different playing positions estimated from the generalised mixed linear model controlling the effects of game location, competition phase, team and opposition strength are presented in Table 2. The comparison on the difference in the mean counts of each action or event between players from different playing positions is presented in the Figure 1.

\subsection{Variables related to goal scoring}

Forwards performed more shots and shots on target than central defenders, full backs and wide midfielders, but showed trivial difference when compared to central midfielders. Defenders (central defenders and full backs) made less shots and shots on target than that of midfielders and forwards, while the difference between full backs and central defenders was trivial. The number of shots and shots on target of central midfielders were higher than those of wide midfielders.

Table 2. Descriptive statistics of technical match performance players of different playing positions estimated from the generalised mixed linear model ( $n$ denotes the number of match observations, results are mean \pm between-player standard deviation, units are counts, except for pass accuracy).

\begin{tabular}{lccccc}
\hline Variable & $\mathrm{CD}(n=3,632)$ & $\mathrm{FB}(n=3,429)$ & $\mathrm{CM}(n=4,044)$ & $\mathrm{WM}(n=1,565)$ & $\mathrm{FW}(n=1,767)$ \\
\hline Shot & $0.67 \pm 1.20$ & $0.52 \pm 1.10$ & $1.41 \pm 1.68$ & $1.11 \pm 1.49$ & $1.59 \pm 1.80$ \\
Shot on target & $0.19 \pm 0.88$ & $0.13 \pm 0.93$ & $0.54 \pm 1.05$ & $0.37 \pm 0.94$ & $0.65 \pm 1.12$ \\
Touch & $58.3 \pm 19.3$ & $57.5 \pm 19.1$ & $55.5 \pm 18.6$ & $59.1 \pm 19.5$ & $51.1 \pm 17.7$ \\
Pass & $42.3 \pm 18.1$ & $40.3 \pm 17.5$ & $38.9 \pm 17.1$ & $43.0 \pm 18.3$ & $34.9 \pm 15.9$ \\
Pass accuracy (\%) & $81.2 \pm 10.3$ & $79.2 \pm 10.1$ & $78.6 \pm 10.1$ & $81.1 \pm 10.3$ & $78.3 \pm 10.1$ \\
Key pass & $0.53 \pm 1.14$ & $0.63 \pm 1.21$ & $0.98 \pm 1.46$ & $0.86 \pm 1.37$ & $1.07 \pm 1.53$ \\
Cross & $0.90 \pm 2.34$ & $1.41 \pm 3.06$ & $1.13 \pm 2.65$ & $0.99 \pm 2.45$ & $1.12 \pm 2.64$ \\
Long ball & $4.36 \pm 3.78$ & $4.02 \pm 3.59$ & $3.17 \pm 3.11$ & $3.94 \pm 3.54$ & $2.12 \pm 2.50$ \\
Through ball & $0.09 \pm 0.99$ & $0.05 \pm 1.53$ & $0.13 \pm 0.91$ & $0.13 \pm 0.91$ & $0.19 \pm 0.90$ \\
Dribble & $0.51 \pm 1.25$ & $0.60 \pm 1.33$ & $0.81 \pm 1.52$ & $0.70 \pm 1.41$ & $0.78 \pm 1.49$ \\
Aerial won & $1.15 \pm 1.74$ & $0.97 \pm 1.61$ & $0.90 \pm 1.56$ & $0.91 \pm 1.56$ & $1.03 \pm 1.65$ \\
Fouled & $0.94 \pm 1.30$ & $1.01 \pm 1.34$ & $1.42 \pm 1.57$ & $1.30 \pm 1.51$ & $1.51 \pm 1.62$ \\
Dispossessed & $0.58 \pm 1.16$ & $0.58 \pm 1.16$ & $1.23 \pm 1.61$ & $1.04 \pm 1.48$ & $1.31 \pm 1.67$ \\
Tackle & $2.15 \pm 1.96$ & $2.46 \pm 2.10$ & $1.89 \pm 1.83$ & $2.26 \pm 2.00$ & $1.41 \pm 1.60$ \\
Interception & $2.13 \pm 1.96$ & $2.11 \pm 1.96$ & $1.52 \pm 1.68$ & $1.82 \pm 1.82$ & $0.98 \pm 1.41$ \\
Clearance & $2.74 \pm 3.22$ & $2.72 \pm 3.20$ & $1.31 \pm 2.11$ & $1.98 \pm 2.63$ & $0.81 \pm 1.73$ \\
Foul & $1.23 \pm 1.40$ & $1.31 \pm 1.44$ & $1.48 \pm 1.53$ & $1.50 \pm 1.54$ & $1.52 \pm 1.54$ \\
Yellow card & $0.21 \pm 0.70$ & $0.18 \pm 0.70$ & $0.18 \pm 0.70$ & $0.20 \pm 0.70$ & $0.17 \pm 0.70$ \\
\hline
\end{tabular}




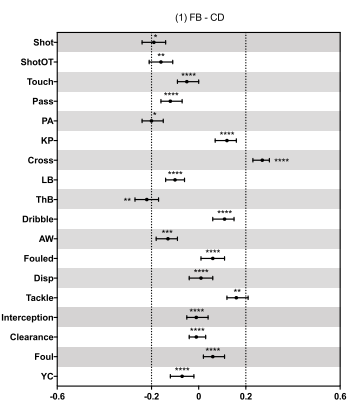

(5) $\mathrm{CM}-\mathrm{FB}$
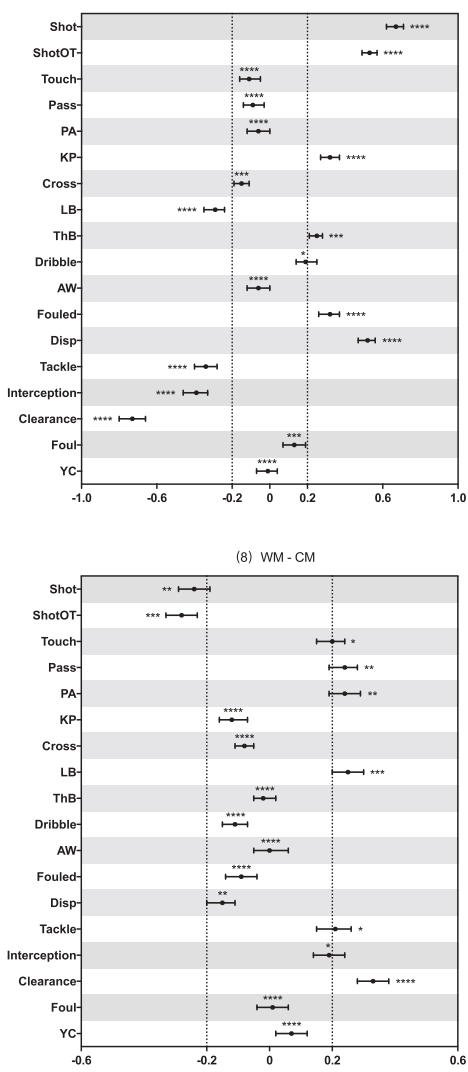
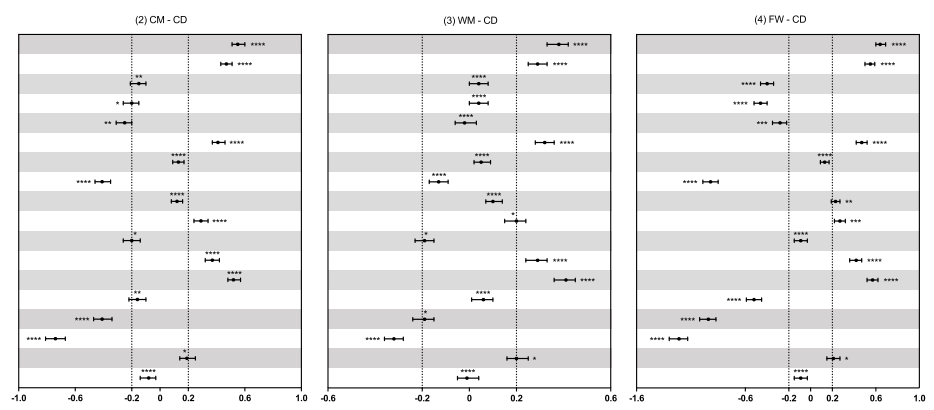

(6) $W M-F B$
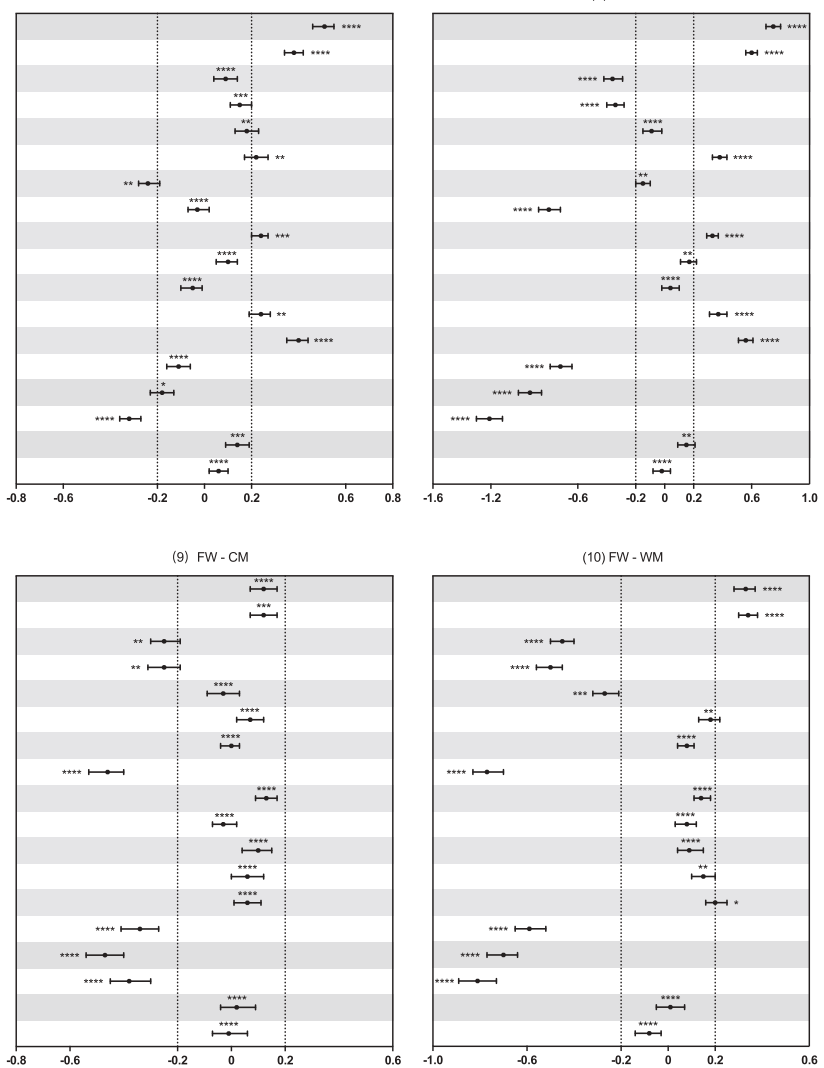

Figure 1. Effect sizes of differences in mean count of each match action or event between players from five playing positions.

Asterisks indicate the likelihood for the magnitude of the true differences in mean as follows: ${ }^{\text {possible; }}{ }^{* *}$ likely; ${ }^{* * *}$ very likely; ${ }^{* * *}$ most likely. Asterisks located in the area between -0.2 and 0.2 denote for trivial differences.

ShotOT = shot on target; $\mathrm{PA}=$ pass accuracy in \%; $\mathrm{KP}=$ key pass; $\mathrm{LB}=$ long ball; $\mathrm{ThB}=$ through ball; $\mathrm{AW}=$ aerial won; Disp = dispossessed; $Y C=$ yellow card.

\subsection{Variables related to passing and organising}

Through ball and cross were the only two variables that showed clear differences between central defenders and full backs, central defenders obtained more through balls and less crosses than that of full backs, while substantial differences between central midfielders and wide midfielders were observed in pass, pass accuracy and 
long ball, wide midfielders had greater number of these three variables compared with central midfielders. Central midfielders only showed the greatest number of dribbles compared to the rest of playing positions in all variables related to passing and organising, whereas only the comparison with central defenders showed clear difference and the differences in the pairwise comparisons with other playing positions were trivial. Forwards had the lowest value of touches, passes, and through balls. Moreover, only cross and aerial won showed trivial differences between forwards and central defenders when considering variables related to passing and organising.

Midfielders (central midfielders and wide midfielders) gained more key passes, fouled and dispossessed than that of defenders (central defenders and full backs). However, no substantial differences were detected in touch and aerial won between defenders and midfielders. In addition, central defenders had more passes, pass accuracy and long balls than central midfielders, full backs performed more crosses than wide midfielders. As for the differences between midfielders (central midfielders and wide midfielders) and forwards, midfielders presented higher number of touches, passes and long balls compared to those of forwards while there were no substantial differences detected in key pass, cross, through ball, dribble, aerial won and fouled. Additionally, midfielders and forwards showed a substantially greater number of key passes, fouled and dispossessed than defenders. When considering the differences between defenders (central defenders and full backs) and forwards, defenders obtained more touches, passes and long balls and less key passes, through balls, dispossessed and fouled than that of forwards, while the differences in cross and aerial won were trivial.

\subsection{Variables related to defending}

There were no substantial differences between central defenders and full backs in all variables related to defending. The number of tackles and clearances of wide midfielders were higher than that of central midfielders and the differences of interception, foul and yellow card were trivial. Forwards had the lowest number of tackles, interceptions and clearances compared with other four playing positions. Additionally, forwards and central midfielders committed more fouls than central defenders.

Only clearance showed substantial differences and yellow card showed trivial differences between defenders (central defenders and full backs) and midfielders (central midfielders and wide midfielders), defenders had more clearances than midfielders. Midfielders (central midfielders and wide midfielders) gained more tackles, interceptions and clearances than that of forwards while no substantial differences were ascertained in foul and yellow card. The comparison between defenders (central defenders and full backs) and forwards showed the similar result that defenders had more tackles, interceptions and clearances than that of forwards but only yellow card showed trivial difference.

\section{Discussion}

The current study identified the differences of technical demands between playing positions in the UEFA Champions League by controlling the effects of situational 
variables (competition stage, match location, team and opponent strength) from a longterm perspective ( 8 seasons). The different positions on the field were characterised by specific technical requirements and position specific key performance indicators were identified in different aspects of match performance (goal scoring, passing and organising, defending).

Our results showed that forwards achieved the greatest number of shots and shots on target, which is similar to the findings reported by Taylor et al. (2004). Moreover, central midfielders showed trivial difference with forwards in the number of shots and shots on target, but showed substantial higher numbers than wide midfielders, which may indicate that central midfielders played a more offensive role in the modern football tactics and made more contributions in the finalisation of the attacking process (Liu et al., 2016a). It is unsurprising that there was a trivial difference between central defenders and full backs in the performance related to goal scoring, and both were worse than midfielders and forwards, which can be easily explained by the similarities in the tactical duties between central defenders and full backs and the differences of positional roles between defenders, midfielders and forwards.

The differences of technical demands related to passing and organising between central defenders and full backs were mainly in through ball and cross. Even though the number of through balls achieved by central defenders was higher than that of full backs, whereas both of them were at a relative lower level than midfielders and forwards due to the position specific duties. Additionally, full backs were observed having higher frequencies in cross than central defenders and even higher than wide midfielders. This finding demonstrates that crossing is a main technical requirement for full backs (Hughes et al., 2012; Van Lingen, 1998), they were required to enter more into the attacking third and sending the ball from wide area into the opponent's area when in possession, while wide midfielders move into central area attracting opponent's defenders inside and creating space for full backs (Bush et al., 2015). The mean value of pass, pass accuracy and long ball of central midfielders not only was lower than that of wide midfielders, but also was lower than that of central defenders. Together with the aforementioned results that the relative better performance of central midfielders in variables related to goal scoring, we found that the central midfielders are moving forward more into the opponent's penalty area in the offensive phases thus they can get more scoring opportunities. This finding partly contrast with research by Liu et al. (Liu et al., 2016a) who presented evidence that good central midfielders in the Spanish professional football league primarily focus on passing and organising duties rather than invading too much into the opponent's area. Furthermore, teams employed more the wide area to seek offensive opportunities thus more passing and organising related actions or events were performed by wide players in their areas (Almeida, Ferreira, \& Volossovitch, 2014). The position specific duties of players on the pitch may once again account for that the forwards obtained the lowest value of touches, passes and through balls and the substantial differences between forwards and central defenders. Unexpectedly, all playing positions showed the same level of performance in aerial won, which can be supported by a previous study that indicated the heading ability is a common technical requirement for all playing positions (Hughes et al., 2012). This result is in contrast with the general consensus that forwards and central defenders are 
those who possess good heading ability. In fact, aerial duel occurs at all over the pitch not just in the attacking/defending third.

Regarding the differences of technical demands related to passing and organising between defenders and midfielders, the number of key pass, fouled and dispossessed of midfielders were higher than those for defenders. A possible reason is that midfielders' position is closer to the opponent's area than defenders and they provide support for forwards by sending key passes, they have to face more defensive pressure due to the higher player intensity thus more fouled and dispossessed were obtained. In addition, the number of pass, pass accuracy and long ball of central defenders were higher than those for central midfielders, which may reveal a trend that central defenders are more involved in the attacking process when team in possession (Bush et al., 2015) and playing the role of launching point to initiate team's offense sending the ball to the forwards in a more direct way (Liu et al., 2016a). Another concern is that central defenders showed higher pass accuracy than central midfielders, which may be probably due to the fact that central defenders faced less defensive pressure from opponent. In the comparison between midfielders and forwards, clear differences were found in touch, pass and long ball and trivial differences were found in dribble, aerial won and fouled, which is consistent with our expectation. However, one noteworthy finding is that trivial differences in the performance between midfielders and forwards were found in key pass, cross and through ball, which may demonstrate that the task of forwards in the attacking area is not only seizing the scoring opportunities provided by teammates, but also creating opportunities for teammates by proper ability of passing and organising. Differences in the performance of passing and organising between defenders and forwards were biggest, especially between central defenders and forwards, which may well be explained by the fact that the biggest differences exist between positional duties of these two field positions.

Concerning the variables related to defending, no substantial differences were found between the performance of central defenders and full backs, which could have been expected. However, wide midfielders had more tackles and clearances than central midfielders, which may suggest that wide midfielders might have more defensive tasks than central midfielders during match play (Almeida et al., 2014), which is consistent with the aforementioned finding that the teams' offense were launched more from the wide area. Generally, defenders demonstrated the highest involvement in defending related actions while forwards presented the lowest and midfielders lay in the middle. Nevertheless, even though forwards achieved the lowest tackles, interceptions and clearances, they committed the most fouls. Combining the fact that the number of fouls committed by wide and central midfielders showed only trivial difference with that of forwards, it may be induced that the defending of modern football starts in opponent's half by the pressing of forwards (Dellal et al., 2011), inventible and necessary fouls are more committed by the midfielders and forwards in areas far away from the defending third of own team in order to delay or interfere opponents' attack. This can be partially supported by the finding that yellow card showed trivial differences among all playing positions.

\section{Conclusions}

It could be concluded that the main findings of the present study reveal that technical demands differ significantly across different playing positions when 
controlling the effects of situational variables of competition stage, match location, team and opponent strength. The biggest difference was found between central defenders and forwards while central defenders and full backs presented the smallest difference. In this study, the basic positional technical demands were identified, which is in line with previous research and in accordance with the specific positional role of each playing position on the pitch. Meanwhile, new trends were also detected. Central defenders involved more in the link-up and attacking process as the launching point of offense. Invading more into the attacking third and sending the ball from wide position to central position (cross) is the main task for full backs during the offensive phase. Central midfielders showed worse performance in passing and organising and better performance in goal scoring than we had expected. Wide midfielders possessed the ability of control the ball and moving more into the opponent's area. Forwards made more contribution in the defence when the possession was lost and they also showed the capacity of creating the scoring opportunities for teammates in the attacking process. The data presented within the present study identified that different playing positions on the field are characterised by specific technical activities and demands, which might provide valuable insights into the trend of modern football match-play and developing individualised conditional training programmes for soccer players.

\section{Acknowledgements}

Authors would like to appreciate the website of "whoscored.com" for the permission to the academic usage of their data.

\section{Disclosure statement}

No potential conflict of interest was reported by the authors.

\section{Funding}

The first author is funded by the China Scholarship Council (CSC) from the Ministry of Education of P.R. China [Grant No. 201608390011]. This work was supported by the Project of Youth Innovative Talents in Colleges and Universities from Department of Education of Guangdong Province [Grant number 2016WQNCX015].

\section{ORCID}

Qing Yi (D) http://orcid.org/0000-0001-8543-1974

Hongyou Liu (D) http://orcid.org/0000-0003-4341-672X

Miguel Ángel Gómez (D) http://orcid.org/0000-0002-9585-3158

\section{References}

Almeida, C. H., Ferreira, A. P., \& Volossovitch, A. (2014). Effects of match location, match status and quality of opposition on regaining possession in UEFA champions league. Journal of Human Kinetics, 41, 203-214. 
Bloomfield, J., Polman, R., \& O’Donoghue, P. (2007). Physical demands of different positions in FA premier league soccer. Journal of Sports Science \& Medicine, 6, 63.

Boone, J., Vaeyens, R., Steyaert, A., Bossche, L. V., \& Bourgois, J. (2012). Physical fitness of elite Belgian soccer players by player position. The Journal of Strength \& Conditioning Research, 26, 2051-2057.

Bush, M., Barnes, C., Archer, D. T., Hogg, B., \& Bradley, P. S. (2015). Evolution of match performance parameters for various playing positions in the english premier league. Human Movement Science, 39, 1-11.

Butterworth, A., O’Donoghue, P., \& Cropley, B. (2013). Performance profiling in sports coaching: A review. International Journal of Performance Analysis in Sport, 13, 572-593.

Carling, C. (2010). Analysis of physical activity profiles when running with the ball in a professional soccer team. Journal of Sports Sciences, 28, 319-326.

Castellano, J., Casamichana, D., \& Lago, C. (2012). The use of match statistics that discriminate between successful and unsuccessful soccer teams. Journal of Human Kinetics, 31, 137-147.

Dellal, A., Chamari, K., Pintus, A., Girard, O., Cotte, T., \& Keller, D. (2008). Heart rate responses during small-sided games and short intermittent running training in elite soccer players: A comparative study. The Journal of Strength \& Conditioning Research, 22, 1449-1457.

Dellal, A., Chamari, K., Wong, D. P., Ahmaidi, S., Keller, D., Barros, R., ... Carling, C. (2011). Comparison of physical and technical performance in European soccer match-play: FA Premier League and La Liga. European Journal of Sport Science, 11, 51-59.

Dellal, A., Wong, D. P., Moalla, W., \& Chamari, K. (2010). Physical and technical activity of soccer players in the French First League-with special reference to their playing position. International SportMed Journal, 11, 278-290.

Di Salvo, V., Baron, R., Tschan, H., Montero, F. C., Bachl, N., \& Pigozzi, F. (2007). Performance characteristics according to playing position in elite soccer. International Journal of Sports Medicine, 28, 222-227.

Gómez, M., Lago, C., \& Pollard, R. (2013). Situational variables. In McGarry, T., O’donoghue, P., \& Sampaio, J. (Eds.), Routledge handbook of sports performance analysis (pp. 259-269). London, UK: Routledge.

Hopkins, W. (2016). SAS (and R) for mixed models. Spreadsheet: Process poisson and logistic repeated measures. Sportscience, 20, iii.

Hopkins, W., Marshall, S., Batterham, A., \& Hanin, J. (2009). Progressive statistics for studies in sports medicine and exercise science. Medicine+ Science in Sports+ Exercise, 41, 3.

Hughes, M., Caudrelier, T., James, N., Redwood-Brown, A., Donnelly, I., Kirkbride, A., \& Duschesne, C. (2012). Moneyball and soccer-an analysis of the key performance indicators of elite male soccer players by position. Journal of Human Sport and Exercise, 7.

Lago-Peñas, C., Casais, L., Dellal, A., Rey, E., \& Domínguez, E. (2011a). Anthropometric and physiological characteristics of young soccer players according to their playing positions: Relevance for competition success. The Journal of Strength \& Conditioning Research, 25, 3358-3367.

Lago-Peñas, C., \& Lago-Ballesteros, J. (2011). Game location and team quality effects on performance profiles in professional soccer. Journal of Sports Science \& Medicine, 10, 465.

Lago-Peñas, C., Lago-Ballesteros, J., Dellal, A., \& Gómez, M. (2010). Game-related statistics that discriminated winning, drawing and losing teams from the Spanish soccer league. Journal of Sports Science \& Medicine, 9, 288.

Lago-Peñas, C., Lago-Ballesteros, J., \& Rey, E. (2011b). Differences in performance indicators between winning and losing teams in the UEFA champions league. Journal of Human Kinetics, 27, 135-146.

Liu, H., Gómez, M.-A., Gonçalves, B., \& Sampaio, J. (2016a). Technical performance and matchto-match variation in elite football teams. Journal of Sports Sciences, 34, 509-518.

Liu, H., Hopkins, W., Gómez, A. M., \& Molinuevo, S. J. (2013). Inter-operator reliability of live football match statistics from OPTA sportsdata. International Journal of Performance Analysis in Sport, 13, 803-821. 
Liu, H., Hopkins, W. G., \& Gómez, M.-A. (2016b). Modelling relationships between match events and match outcome in elite football. European Journal of Sport Science, 16, 516-525.

Liu, H., Yi, Q., Giménez, J.-V., Gómez, M.-A., \& Lago-Peñas, C. (2015). Performance profiles of football teams in the UEFA champions league considering situational efficiency. International Journal of Performance Analysis in Sport, 15, 371-390.

Mao, L., Peng, Z., Liu, H., \& Gómez, M. A. (2016). Identifying keys to win in the Chinese professional soccer league. International Journal of Performance Analysis in Sport, 16, 935947.

Mohr, M., Krustrup, P., \& Bangsbo, J. (2005). Fatigue in soccer: A brief review. Journal of Sports Sciences, 23, 593-599.

Phillips, K. E., \& Hopkins, W. G. (2017). Performance relationships in timed and mass-start events for elite omnium cyclists. International Journal of Sports Physiology \& Performance, 12, 628-633.

Reilly, T., Bangsbo, J., \& Franks, A. (2000). Anthropometric and physiological predispositions for elite soccer. Journal of Sports Sciences, 18, 669-683.

Taylor, J. B., Mellalieu, S. D., \& James, N. (2004). Behavioural comparisons of positional demands in professional soccer. International Journal of Performance Analysis in Sport, 4, 81-97.

Vaeyens, R., Lenoir, M., Williams, A. M., \& Philippaerts, R. M. (2008). Talent identification and development programmes in sport. Sports Medicine, 38, 703-714.

Vale, P., Ramos, A., Salgado, B., Correia, P., Martins, P., Brito, J., ... Rebelo, A. (2009). Differences in technical skill performance of Portuguese junior soccer players according to competitive level and playing position. Paper presented at the International Research in Science and Soccer The Proceedings of the First World Conference on Science and Soccer, Liverpool, UK.

Van Lingen, B. (1998). Coaching soccer: The official coaching book of the dutch soccer association. Spring City, PA: Reedswain.

Wiemeyer, J. (2017). Who should play in which position in soccer? Empirical evidence and unconventional modelling. International Journal of Performance Analysis in Sport, 3, 1-18. 